\title{
Research on Signaling Mechanism for Marketization Recruitment of Managers in Mixed Ownership Enterprises
}

\author{
Ping Sun \\ Business School, Chengdu University, Chengdu, China \\ sunping@cdu.edu.cn
}

Keywords: Mixed ownership enterprise, Marketization Recruitment, Manager, Ability, Signaling.

\begin{abstract}
Under the background of the gradual implementation of the professional manager system in mixed ownership enterprises and marketization recruitment of managers by the board of directors in China, exploratory research on marketization recruitment mechanism of managers in mixed ownership enterprises needs theoretical guidance and support from the related theories of identification scheme and mechanism. Due to the unobservability of manager ability and manager's behavior of hiding information, problems of manager selection can only be sloved through the identification of manager ability information. By applying the approach of signaling in information economics, this study explores the feasibility and effectiveness of the business plans submitted by managers in mixed ownership enterprises as a non-educational signal to transfer the information on manager ability, so as to provide a theoretical ground and reference for the establishment of marketization recruitment mechanism of managers in mixed ownership enterprises.
\end{abstract}

\section{混合所有制企业经理的市场化选聘的信号传递机制研究}

\author{
孙平 \\ 成都大学商学院, 成都, 四川, 中国 \\ sunping@cdu.edu.cn
}

关键词：混合所有制企业; 市场化选聘; 经理; 能力; 信号传递

中文摘要. 在中国对混合所有制企业逐步推行职业经理人制度, 董事会按市场化方式选聘经 理的背景下, 对混合所有制企业经理市场化选聘机制的探索急需新的识别机制设计相关研究 的理论指导。经理能力的不可观察性和经理隐藏信息的行为使得只能通过经理能力信息的识 别来解决经理的选择问题。本文运用信息经济学的信号传递方法, 探讨经理提交的商业企划 书作为传递有关混合所有制企业经理能力信息的非教育信号机制的可行性及有效性，为建立 混合所有制企业经理的市场化选聘机制提供理论依据和参考建议。

\section{1. 引言}

党的十八届三中全会做出的《中共中央关于全面深化改革若干重大问题的决定》及 2015 年9月国务院正式出台的《关于国有企业发展混合所有制经济的意见》中明确提出将混合所有 制经济作为国有企业改革的重要方向，通过混合所有制解决国有企业的效率问题，混合所有 制改革被确立为当前国企深化改革的重大战略和基本模式。李维安认为在国企改革中引入混 合所有制，将国有资本的资本优势与民营资本的灵活市场机制优势合二为一，从而产生 “1 $1>2 ”$ 的治理效果 ${ }^{[1]}$ 。杨瑞龙认为以混合经济为突破口的国有企业改革需要国有企业分类改 
革战略、国有资产管理与经营体制、国有资本退出路径、混合所有制企业治理结构、职业经 理人制度等措施的统筹安排才能取得实质性效果 ${ }^{[2]}$ 。

经理是企业经营决策的制定和执行者, 其在企业的发展中起着特殊而又非常重要的作用。 经理能力的高低很大程度上直接决定了企业的绩效。经理选择是影响股东、供应商、顾客、 公众和政府等利益相关者对企业未来发展判断的重要因素, 因此也直接关系着企业的市场价 值。在我国国有企业的混合所有制改革中，经理选择对于混合所有制企业的发展甚至生存都 起着至关重要的作用。《关于深化国有企业改革的指导意见》明确提出“推行职业经理人制度, 实行内部培养和外部引进相结合, 畅通现有经营管理者与职业经理人身份转换通道, 董事会 按市场化方式选聘和管理职业经理人，合理增加市场化选聘比例，加快建立退出机制。”市场 化选聘的经理在混合所有制企业中面临完全不同的产权结构和公司治理结构。企业的混合所 有制是指既有国有产权又有非国有产权，形成国有资本和非国有资本“交叉持股”、“相互融合” 的股权结构，即国企允许民营资本参股和民企允许国有资本参股，公司治理结构中即有国有 股东又有非国有股东的控制权 ${ }^{[3-4]}$ 。与传统的国有企业相比, 混合所有制企业对经理的选择有 着更大的资源和资本优势，有着更大选择空间和自由度。由于经理的能力是私人信息，同时 他有隐藏能力信息的动机。经理能力的不可观察性和经理隐藏信息的行为使得只能通过经理 能力信息的识别来解决经理的选择问题。本文运用信息经济学的信号传递方法, 探讨混合所 有制企业经理提交的商业企划书作为传递经理能力信息的非教育信号机制的可行性及有效 性，为建立混合所有制企业经理的市场化选聘机制提供理论依据和参考建议。

\section{2. 文献评述}

信号传递是指在协议形成之前，知情的一方通过个人行为披露私人信息。由于经理是通 过企业外部的市场机制传递其能力信息，所以信号传递作为一种识别经理能力信息的方法， 又称为外部市场识别机制。

信号传递理论的开创者Spence用“信号传递博奕”建立了一个劳动力就业市场模型。在这 个模型中，劳动者的生产能力大小是私人信息，劳动者知道自己的能力，雇主不知道，市场 通过观测劳动者的受教育程度, 并以文凭为标准支付其工资。受教育程度是劳动者发出的一 个有关能力的信号 ${ }^{[5]}$ 。职业经理人市场可以看成一个特殊的劳动力市场, 在经理的任用过程 中, 同样存在着文凭标准现象, 即以受教育程度来间接反映经理能力的大小, Spence的模型 对职业经理人市场如何甄别代理人的能力也是有一定的解释作用的。但是，职业经理人市场 毕竟有其特殊之处, 经理能力与一般劳动者能力有着明显的差异, 用于识别经理能力信息的 信号也不能简单只是受教育程度。Fama提出可以通过观察企业的市场价值、利润等指标来推 测企业家才能的高低 ${ }^{[6]}$ 。Zwiebel指出, 在代理人能力信息不对称的情况下, 相对业绩评价方 法 (CPI) 将诱导出高能力和低能力代理人比平均能力水平代理人更多的创新行为, 从而CPI 在隐性激励过程中可以用于评价经理能力的高低 ${ }^{[7]}$ 。张维迎提出一个“经营能力一个人财富” 模型, 该模型指出企业家以拥有的个人财富向市场传递企业家能力的信息 ${ }^{[8]}$ 。魏明建立企业 家的人力资本模型, 通过企业家人力资本的积累过程和市场配置的动态过程来描述企业家人 力资本的显示机制 ${ }^{[9]}$ 。朱平辉指出反映“企业家能力”的可信的“信号”包括他所接受的教育程 度, 和他在企业家市场上从事经营活动的时间 ${ }^{[10]}$ 。石树琴通过风险企业家信号传递模型指出 在风险投资的开拓阶段，风险投资家和风险企业家之间通过经营计划书来传递信息 ${ }^{[11]}$ 。本文 在已有研究的基础上, 以经理策划和呈现的商业企划书水平代表传递经理能力信息的非教育 信号建立经理能力信号传递模型，深入讨论其可行性并分析影响其有效性的因素。 


\section{3. 经理能力信号传递模型}

\section{1 假设}

假设 1 : 经理能力 $a$ 为离散变量, 有两个可能的值, 分别为 $a_{1}$ 和 $a_{2}, a_{1}$ 代表低能力, $a_{2}$ 代 表高能力, $a_{1}<a_{2}$ 。

假设 2 : 经理能力 $a$ 是经理的私人信息, 职业经理人市场不能直接观察到经理的能力信息, 职业经理人市场只知道 $a_{1}$ 和 $a_{2}$ 的概率均为 $1 / 2$ 。

假设 3 : 经理在与混合所有制企业签约之前选择商业企划书水平 $s$ ，作为传递有关经理能 力信息的信号, $s$ 是一个连续变量, 且 $s \in[0, \bar{s}]$, 其中, $\bar{s}$ 为常数, 代表商业企划书的最高水 平。

假设 $4: \pi$ 是能力 $a$ 和信号水平 $s$ 的函数, 即 $\pi=f(a, s), \pi$ 代表经理对混合所有制企业产出 的期望贡献, 简称为经理期望贡献。用下式简化表示 $\pi$ 与 $a 、 s$ 之间的函数关系为:

$$
\pi=f(a, s)=r_{1} a+r_{2} s+r_{3} a s\left(r_{1}, r_{2}, r_{3} \geq 0\right)
$$

其中, $a s$ 代表经理能力与商业企划书水平之间互动对产出的作用, $r_{1}, r_{2}, r_{3}$ 分别代表 $a$, $s$, as 对经理期望贡献的边际影响。

由于假设经理能力 $a$ 为离散变量, 只取低能力 $a_{1}$ 和高能力 $a_{2}$ 两个可能的值, 经理的期望贡 献函数 (1) 可以进一步表示为:

$$
\pi=\left\{\begin{array}{l}
r_{1} a_{1}+r_{2} s+r_{3} a_{1} s, a=a_{1} \\
r_{1} a_{2}+r_{2} s+r_{3} a_{2} s, a=a_{2}
\end{array}\left(r_{1}, r_{2}, r_{3} \geq 0\right)\right.
$$

假设 5: $\frac{\partial U}{\partial \omega}>0, \frac{\partial^{2} U}{\partial \omega^{2}} \leq 0, \frac{\partial U}{\partial s}<0, \frac{\partial^{2} U}{\partial s^{2}}<0, \frac{\partial U_{1}}{\partial s}<\frac{\partial U_{2}}{\partial s}$, 其中, $U=U_{i}(\omega, s)$ 代表能力为 $a_{i}$ 的 经理的效用函数（ $i=1,2 ） ， \omega$ 代表经理预期将得到的收入。

由假设 5 可知，收入带来正效用，且边际效用递减（或不变）; 由于经理策划和呈现商业 企划书需要付出成本, 所以商业企划书的策划和呈现会给经理带来负效用, 且边际成本递增; 低能力经理的商业企划书的边际成本高于高能力经理的商业企划书的边际成本。从 $s$ 和 $\omega$ 构成 的无差异曲线上看, 假设 5 意味着低能力经理的无差异曲线和高能力经理的无差异曲线的斜率 都为正, 且低能力经理的无差异曲线处处陡于高能力经理的无差异曲线, 二者只有一个交点 (“单交叉条件”) [12]，见图1。

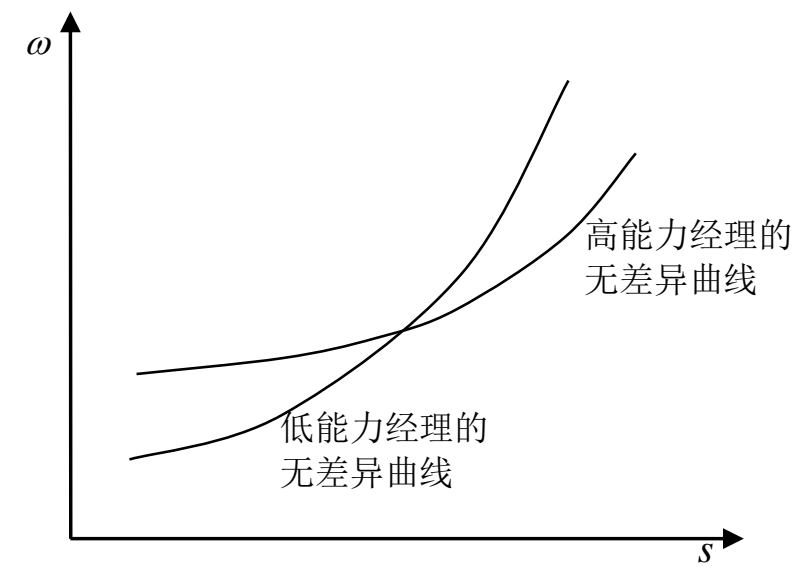

图1 无差异曲线和单交叉条件

假设6: 当观察到经理的商业企划书水平为 $s$ 时, 职业经理人市场认为经理属于低能力的 后验概率为 $\mu(s)=\mu\left(a=a_{1} \mid s\right)$, 属于高能力的后验概率为 $1-\mu(s)=1-\mu\left(a=a_{1} \mid s\right)$ 。 


\section{2 建模及均衡解}

在信息不对称情况下, 即经理能力是经理的私人信息, 不能直接观察到经理的能力信息, 而商业企划书水平作为传递有关经理能力信息的信号, 职业经理人市场通过它间接观察经理 的能力信息。此时，精炼贝叶斯均衡（不完全信息动态均衡）为:

存在一个预期的收入函数 $\omega(s)$, 一个商业企划书水平 $s^{*}(a)$, 和一个后验概率 $\mu(s)$, 使得:

(1) 给定 $\omega(s), s^{*}$ 最大化 $U_{i}(\omega(s), s)$;

(2) $\omega\left(s^{*}\right)=\mu\left(s^{*}\right)\left(r_{1} a_{1}+r_{2} s^{*}+r_{3} a_{1} s^{*}\right)+\left(1-\mu\left(s^{*}\right)\right)\left(r_{1} a_{2}+r_{2} s^{*}+r_{3} a_{2} s^{*}\right)$;

(3) $\mu(s)$ 与贝叶斯规则相一致。

条件（1）称为激励相容约束或“自选择约束”（self-selection constraint）：给定预期的收 入函数, 能力为 $a_{i}$ 的经理将选择使自己的效用函数最大化的商业企划书水平 $s^{*}\left(a_{i}\right)(i=1,2)$; 条件（2）是“参与约束”: 在均衡时, 经理的收入等于经理贡献的期望值（竞争性假设）; 条 件 (3) 是贝叶斯条件 ${ }^{[12]}$ 。

信息对称情况下的均衡解：在信息对称情况下，即在职业经理人市场可以直接观察到经 理能力信息的情况下, $\mathrm{A}$ 和 $\mathrm{B}$ 分别是低能力经理和高能力经理的均衡点。低能力经理选择商业 企划书水平 $\hat{s}_{1}$, 得到收入 $\omega=r_{1} a_{1}+r_{2} \hat{s}_{1}+r_{3} a_{1} \hat{s}_{1}$; 高能力经理选择商业企划书水平 $\hat{s}_{2}$, 得到 收入 $\omega=r_{1} a_{2}+r_{2} \hat{s}_{2}+r_{3} a_{2} \hat{s}_{2}$, 见图2。

信息不对称情况下的均衡解：在信息不对称情况下，即在职业经理人市场不能直接观察 到经理能力信息的情况下, $\mathrm{A}$ 和 $\mathrm{E}$ 分别是低能力经理和高能力经理的均衡点。低能力经理选择 $\hat{s}_{1}$, 高能力经理选择 $s_{2}{ }^{*}$; 企业认为 $\hat{s}_{1}$ 的是低能力, 给予 $\omega=r_{1} a_{1}+r_{2} \hat{s}_{1}+r_{3} a_{1} \hat{s}_{1}, s_{2}{ }^{*}$ 的是高 能力, 给予 $\omega=r_{1} a_{2}+r_{2} s_{2}{ }^{*}+r_{3} a_{2} s_{2}{ }^{*}$, 见图2。

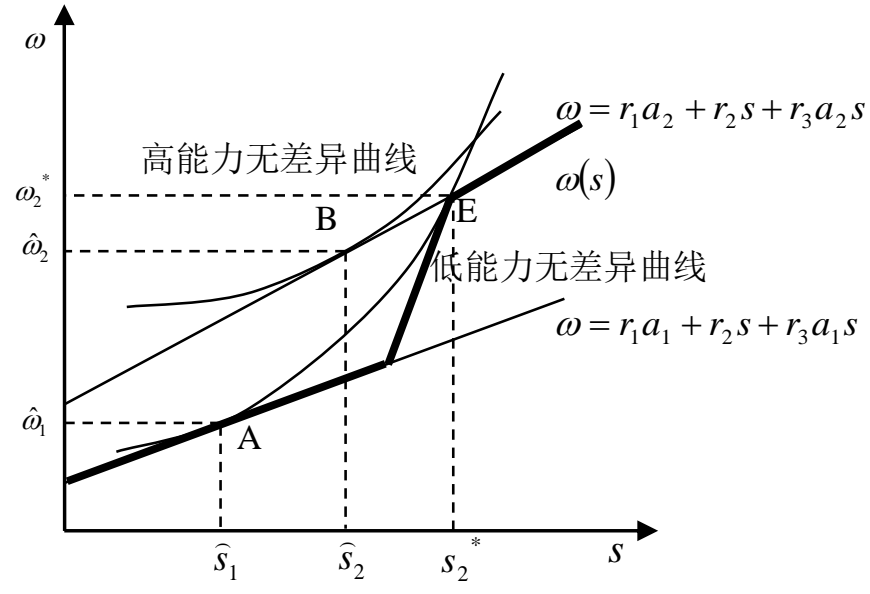

图2 不完全信息下的均衡

\section{4. 信号传递机制有效性分析}

将信息对称情况下的均衡解与信息不对称情况下的均衡解相比较, 在信息不对称情况下, 低能力的经理选择的最优商业企划书水平与信息对称情况下相同, 即 $s_{1}=\hat{s}_{1}$, 但高能力的经 理选择的最优商业企划书水平高于信息对称情况下的最优商业企划书水平，即 $s_{2}{ }^{*} \geq \hat{s}_{2}$ 。记 $\Delta s=s_{2}{ }^{*}-\hat{s}_{2}$, 这种信息不对称情况下的最优水平与信息对称情况下的最优水平的偏离就是 通过商业企划书水平将不同能力的经理区别开的信息成本。这种信息成本的大小决定了商业 企划书水平作为传递有关混合所有制企业经理能力信息的信号的有效性。信息成本越小，商 业企划书越能有效传递经理的能力信息，越被职业经理人市场和混合所有制企业所认可和采 用; 信息成本越大, 商业企划书越不能有效传递经理的能力信息, 越不被职业经理人市场和 混合所有制企业所认可和采用。 
假定能力为 $a_{i}$ 的经理的效用函数为 $U_{i}(\omega, s)=-e^{-\omega+\frac{b_{i}}{2} s^{2}}\left(i=1,2, b_{1}>b_{2}>0\right)$, 其中, $\frac{b_{i}}{2} s^{2}$ 代表能力为 $a_{i}$ 的经理策划和呈现商业企划书的成本, $b_{i}$ 代表成本系数: $b_{i}$ 越大, 同样的商 业企划书水平 $s$ 带来的负效用越大。假定 $b_{1}>b_{2}>0$ 是为了满足假设 5 中的 $\frac{\partial U_{1}}{\partial s}<\frac{\partial U_{2}}{\partial s}$, 即低能 力经理的商业企划书的边际成本高于高能力经理的商业企划书成本。

在信息对称情况下，企业之间的竞争使得经理的均衡收入等于经理的贡献： $\omega_{1}=\pi_{1}=$ $r_{1} a_{1}+r_{2} s+r_{3} a_{1} s, \omega_{2}=\pi_{2}=r_{1} a_{2}+r_{2} s+r_{3} a_{2} s$ 。根据最优化条件, 求解A点:

$$
\left\{\begin{array}{c}
\hat{s}_{1}=\frac{r_{2}+r_{3} a_{1}}{b_{1}} \\
\widehat{\omega}_{1}=r_{1} a_{1}+\frac{\left(r_{2}+r_{3} a_{1}\right)^{2}}{b_{1}}
\end{array}\right.
$$

根据最优化条件，求解 $\mathrm{B}$ 点：

$$
\left\{\begin{array}{c}
\hat{s}_{2}=\frac{r_{2}+r_{3} a_{2}}{b_{2}} \\
\widehat{\omega}_{2}=r_{1} a_{2}+r_{2} \frac{r_{2}+r_{3} a_{2}}{b_{2}}+r_{3} a_{2} \frac{r_{2}+r_{3} a_{2}}{b_{2}}
\end{array}\right.
$$
为:

由式 (3) 进一步可知, 图2中与 $\omega=r_{1} a_{1}+r_{2} s+r_{3} a_{1} s$ 相切于 $\mathrm{A}$ 点的低能力无差异曲线 $\hat{U}_{1}$

$$
\omega=\frac{b_{1}}{2} s^{2}+r_{1} a_{1}+\frac{\left(r_{2}+r_{3} a_{1}\right)^{2}}{2 b_{1}}
$$

低能力无差异曲线 $\hat{U}_{1}$ 与 $\omega=r_{1} a_{2}+r_{2} s+r_{3} a_{2} s$ 相交于 $\mathrm{E}$ 点, 联立求解下式

$$
\left\{\begin{array}{c}
\omega=\frac{b_{1}}{2} s^{2}+r_{1} a_{1}+\frac{\left(r_{2}+r_{3} a_{1}\right)^{2}}{2 b_{1}} \\
\omega=r_{1} a_{2}+r_{2} s+r_{3} a_{2} s
\end{array}\right.
$$

可得E点:

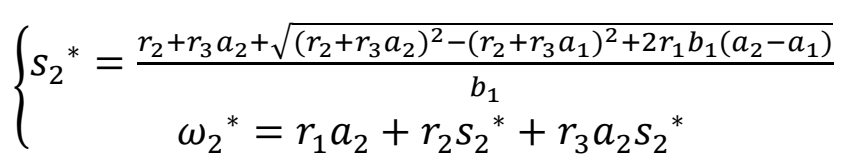

由式（4）和式（6），可得:

$$
\Delta s=s_{2}^{*}-\hat{s}_{2}=\frac{b_{2} \sqrt{\left(2 r_{2} r_{3}+r_{3}{ }^{2} a_{2}+r_{3}^{2} a_{1}+2 r_{1} b_{1}\right)\left(a_{2}-a_{1}\right)}-\left(b_{1}-b_{2}\right)\left(r_{2}+r_{3} a_{2}\right)}{b_{1} b_{2}}
$$

进一步假设 $b_{i}=\frac{1}{a_{i}}(i=1,2)$, 则式 $(7)$ 变为:

$$
\Delta s=s_{2}{ }^{*}-\hat{s}_{2}=a_{1} \sqrt{\left(2 r_{2} r_{3}+r_{3}{ }^{2} a_{2}+r_{3}{ }^{2} a_{1}+\frac{2 r_{1}}{a_{1}}\right)\left(a_{2}-a_{1}\right)}-\left(a_{2}-a_{1}\right)\left(r_{2}+r_{3} a_{2}\right)
$$

由式 (8) 可知, 通过商业企划书水平将不同能力的经理区别开的信息成本 $\Delta s$ 的大小，即 商业企划书水平作为传递有关混合所有制企业经理能力信息的信号的有效性与参数 $r_{1}, r_{2}, r_{3}$, $a_{1}, a_{2}$ 有关。

命题 1: $\frac{\partial \Delta s}{\partial r_{1}}>0$ 。即经理的能力对经理期望贡献的边际影响越大, 通过商业企划书水平将 不同能力的经理区别开的信息成本会越大，相应的信号传递的有效性会越低。

解释命题 1: 当经理对混合所有制企业产出的贡献受经理能力的影响增大时, 在同样的商 业企划书水平 $\hat{s}_{2}$ 情况下, 高能力经理增加的企业产出贡献量大于低能力经理增加的企业产出 贡献量。此时, 低能力经理更有动力模仿高能力经理, 即策划和呈现与高能力经理同等水平 的商业企划书, 为了实现高能力经理与低能力经理的分离需要更高的商业企划书水平。从而, 
将不同能力的经理区别开的信息成本增大，通过商业企划书水平识别混合所有制企业经理能 力信息的可行性和传递有关经理能力信息的有效性降低，这类职业经理人市场和混合所有制 企业越不会认可和采用商业企划书作为识别经理能力信息的手段。

命题 2: $\frac{\partial \Delta s}{\partial r_{2}}<0$ 。即商业企划书的水平对经理期望贡献的边际影响越小, 通过商业企划书 水平将不同能力的经理区别开的信息成本会越大，相应的信号传递的有效性会越低。

解释命题 2: 当经理对混合所有制企业产出的贡献受商业企划书水平的影响减小时, 由于 假设低能力经理的商业企划书的边际成本高于高能力经理的商业企划书的边际成本，此时， 商业企划书水平 $s_{2}$ 的减少量大于商业企划书水平 $s_{2}{ }^{*}$ 的减少量。从而, 将不同能力的经理区别 开的信息成本增大，通过商业企划书水平识别混合所有制企业经理能力信息的可行性和传递 有关经理能力信息的有效性降低，这类职业经理人市场和混合所有制企业越不会认可和采用 商业企划书作为识别经理能力信息的手段。

\section{5. 结论建议}

本文关于商业企划书水平作为传递有关混合所有制企业经理能力信息的信号的可行性及 有效性讨论, 也适用于其它信号传递机制, 从而为建立混合所有制企业经理的市场化选聘机 制提供理论依据和参考建议。

1. 通过市场化选聘的信号传递机制识别混合所有制企业经理能力信息是否可行关键在 于在该传递机制下不同能力的经理发出同样信号的成本是否不同。如果在该传递机制下低能 力经理要发出与高能力经理同样的信号，需要付出更大的成本，则这种传递机制可行。

2. 通过市场化选聘的信号传递机制传递有关混合所有制企业经理能力信息的有效性由 通过该传递机制将不同能力的经理区别开的信息成本的大小决定。信息成本越小，该传递机 制越能有效传递经理的能力信息; 信息成本越大, 该传递机制越不能有效传递经理的能力信 息。

3. 市场化选聘的信号传递机制将不同能力的混合所有制企业经理区别开的信息成本的 大小与经理的能力和该信号的水平对经理期望贡献的边际影响有关。当经理的能力对混合所 有制企业产出的影响越大，通过该传递机制传递有关经理能力信息的有效性会越低，职业经 理人市场和混合所有制企业越不会认可和采用该传递机制。当该信号的水平对混合所有制企 业产出的影响越小，通过该传递机制传递有关经理能力信息的有效性会越低，职业经理人市 场和混合所有制企业越不会认可和采用该传递机制。混合所有制企业经理的市场化选聘的信 号传递机制的有效性需要重点考虑经理的能力和该信号的水平对混合所有制企业产出的影 响。

\section{致谢}

本文为教育部人文社会科学研究一般项目《混合所有制企业多重委托代理冲突的协同治 理研究》(项目编号: 17YJA630088)的阶段性成果之一。

\section{References}

[1] W. A. Li, Deepen the reform of state-owned enterprises and develop mixed ownership, Nankai Business Review, vol. 17 (3), pp. 110-111, 2014.

[2] R. L. Yang, Promoting the reform of state-owned enterprises with mixed economy as a breakthrough, Reform, (5), pp. 19-22, 2014. 
[3] J. B. Li, G. Y. Wang, S. Q. Li, and X. L. Yin, Behavioral game between state-owned capital and non-state-owned capital in mixed ownership reform-evidences from lab experiment, China Industrial Economic, (6), pp. 109-126, 2016.

[4] Y. Hao and L. T. Gong, State and private non-controlling shareholders in SOEs and private firms, and firm performance, Economic Research Journal, (3), pp. 122-135, 2017.

[5] M. Spence, Job market signaling, Quarterly Journal of Economics, vol. 87, pp. 355-374, 1973.

[6] E. Fama, Agency problem and the theory-of the firm. Journal of Political Economy, vol. 88, pp. 288-307, 1980.

[7] J. Zwiebel, Corporate conservation and relative compensation. Journal of Political Economy, vol. 103, pp. 1-25, 1995.

[8] W. Y. Zhang, An Entrepreneurial / Contractual Theory of the Firm. Shanghai: Shanghai People's Publishing House, 2001.

[9] M. Wei, The self-selection and market-disposition of entrepreneur human capital - The theoretical pattern of human capital of entrepreneur and its connotations. Nankai Business Review, vol. 7 (2), pp. 56-62, 2004.

[10]P. H. Zhu, The screening mechanism of entrepreneur ability. Economic Issues in China, vol. (5), pp. 24-31, 2002.

[11]S. Q. Shi, A brief discussion on the model and application of signaling and screening. Journal of Fudan University (Natural Science), vol. 42 (2), pp. 246-252, 2003.

[12]W. Y. Zhang, Game Theory and Information Economics. Shanghai: Shanghai People's Publishing House, 2004. 